\title{
Human Performance Assessment of a Single Air Traffic Controller Conducting Multiple Remote Tower Operations
}

\author{
Peter Kearney ${ }^{1},{ }^{*}$ Wen-Chin Li $^{2}$, Jingyi Zhang ${ }^{3}$, Graham Braithwaite ${ }^{2}$ and Lei Wang ${ }^{3}$ \\ ${ }^{1}$ Irish Aviation Authority, 11-12 D’olier Street, Dublin, Ireland \\ ${ }^{2}$ Safety and Accident Investigation Centre, Cranfield University, U.K. \\ ${ }^{3}$ Flight Technology College, Civil Aviation University of China, Tianjin, China
}

\begin{abstract}
The innovative concept of Multiple Remote Tower Operations (MRTO) can maximize cost savings by applying video panorama-based remote tower working positions, which can facilitate fewer Air Traffic Controllers (ATCO) to provide the Air Traffic Services (ATS) function for more airports. Five subject-matter experts, qualified remote tower ATCOs, participated in this research by applying the Human Error Template (HET) and comparing workload between physical tower operations and MRTO using NASA-TLX (Task Load Index). The results demonstrate that augmented visualization provided sufficient technical support for a single ATCO to perform tasks originally designed to be performed by four ATCOs, however, the demands of the associated multiple tasks induced significant workload. There were significant differences in ATCOs' mental demand, temporal demand, effort and frustration between MRTO and physical tower operations. This innovative technology may induce Human-Computer Interaction (HCI) issues that impact ATCO's perceived workload. This creates a need for further research on how to manage ATCO's workload in a multiple remote tower environment. This research provided scientific evidence that MRTO can achieve the objectives of Single European Sky Air Traffic Management Research program. The findings can be applied to both ATCO training design and remote tower system design.
\end{abstract}

Keywords: Cost-Efficiency; Digital Aviation; Human Performance; Multiple Remote Tower Operations; Perceived Workload 


\section{Introduction}

Initial development of remote tower operations commenced at the end of last century and was designed as a system to permit Air Traffic Services (ATS) to be delivered remotely without direct observation from a local tower. The operational characteristics of remote tower operations differ profoundly from traditional physical tower operations. Digital panoramacameras can be placed on the airfield providing Air Traffic Controller's (ATCO) with realtime enhanced images through augmented visualization functions from advanced technologies. The development of augmented visualization technology has significantly changed the traditional Air Traffic Management (ATM) system and ATCOs' task performance. Under continued pressure from economic regulators to improve Air Navigation Service Provider (ANSP) cost-efficiency, the concept of single tower operations evolved to consider the possibility of Multiple Remote Tower Operations (MRTO) by applying video panoramabased remote tower working positions, which permit less controllers to fulfil the ATM tasks to two or more airports (Kearney \& Li, 2018). The Single European Sky (SES) initiative is expected to increase safety, capacity and reduce costs in order to meet the growing demand for aircraft operations in Europe (Eurocontrol, 2014). An innovative strategy to achieve these objectives is MRTO provided from a remote location from the airports under control. The application of panorama-video cameras enables ATCOs' visually monitor aircraft approaching and departing from the airports under control by video-link from a Remote Tower Centre (RTC). The Out of the Window (OTW) screens function with Pan-Tilt-Zoom (PTZ) cameras permits dynamic object detection, recognition, and identification, permitting the systems to meet the requirements and certification processes of regulators (Fürstenau, Mittendorf, \& Friedrich, 2014). The advanced technology of MRTO also created some human performance safety concerns in relation to Human-Computer Interaction (HCI) and mental workload, as MRTO expected one ATCO to complete the tasks originally managed by four ATCOs. The research questions are to evaluate the human performance and safety issues on MRTO. 


\section{The Innovative Concept of MRTO}

MRTO is also applicable to large airports, in some cases potentially as the primary tower and in others as a fully functioning contingency or backup system. MRTO will require changed procedures and standards from those prescribed in the International Civil Aviation Organization Doc 4444 (ATM) and Eurocontrol's Manual for Aerodrome Flight Information Services (Eurocontrol, 2015). Differences between Air Traffic Control (ATC) provision from a Remote Tower Control (RTC) compared to traditional physical towers requires careful consideration and in-depth assessment to validate human performance capabilities as MRTO involves increases cognitive demands for a single ATCO performing several ATCOs' tasks (Hollan, Hutchins, \& Kirsh, 2000; Li, Kearney, Braithwaite, \& Lin, 2018).

\subsection{New technology induced new HCI challenges}

Air traffic growth in recent years has highlighted deficiencies in infrastructure and airspace capacities resulting in increasing delays to aircraft and passengers. In response to this SES was established with the following objectives - improve safety, reduce the cost to airspace users, and lessen the environmental impacts (Eurocontrol, 2015). ATCOs' visual search whether in radar display or an aerodrome control tower is critical for maintaining Situation Awareness (SA), and their attention distributions can be significantly influenced by the surrounding environment, equipment eco-system and human machine interface design at the Controller Working Position (CWP). In order to achieve an understanding of the effects of different design on cognitive function, it is necessary to apply a holistic approach which includes a comprehensive assessment of human performance on MRTO (Lafond et al., 2009). The objectives of MRTO are to bring capabilities to fit the SES high-level expectations, to enhance system contingency, to enhance ATCO's SA and at least maintain the same level of safety as per traditional towers (Eurocontrol, 2014). The layout of CWP including Electric Flight Strips (EFS), Radar Data Processing (RDP), Voice Communication System (VCS) and OTW screens 
on the remote tower module compared with traditional physical tower consoles shown as figure 1. The innovative designs of remote tower module can impact an ATCO's cognitive processes in terms of attention distribution, SA and decision-making.

[Figure 1 here]

\section{2 Augmented Vision Facilitate ATCO's Monitoring Performance}

ATCOs have to constantly shift attention between outside views and ATM systems which generates workload and accumulates head-down time (Pinska, 2006). Both workload and headdown issues can be resloved by augmented vision design of OTW by superimposing traffic information and weather conditions on the airfield displays (Fürstenau \& Schmidt, 2016; Schmidt, Rudolph, \& Fürstenau, 2016). Human operator's SA and task performance can be significantly improved and cognitive workload can be reduced by appropriate human-cantered design (Laois \& Giannacourou, 1995; Tobaruela et al., 2014; Wickens \& Hollands, 2000). However, inappropriate design of automation can present many disadvantages and create potential system risks leading to accident/incidents, including loss of SA, and placing the human operators outside of the system control loop (Durso, Truitt, Hackworth, Crutchfield, \& Manning, 1998; Endsley, 1995). Augmented visualization design is to enhance ATCO’s SA, using human information-processing models, ATCO's visual behaviours provide an opportunity to investigate the relationship between ATCOs' SA and task performance (Kearney $\& \mathrm{Li}, 2018$ ). Eye scan pattern is one of the most powerful methods for assessing human beings' cognitive processes (Ahlstrom \& Friedman-Berg, 2006). ATCOs have to maintain SA to detect dynamic targets including aircraft in the air, vehicles on the ground at airfields and other hazards such as birds. Effective monitoring performance is foreseen as the most promising way to increase capacity and safety within ATS (Beier \& Gemperlein, 2004). Eye movement patterns 
provide an insight into the ATCO's cognitive information processing through their $\mathrm{HCI}$ on the remote tower operations ( Komogortsev \& Karpov, 2013; Yu, Li, Wang, Braithwaite, \& Greaves, 2016).

\section{3 Monitoring Performance and Perceived Workload on Task Performance}

Air traffic activities are constantly evolving with different traffic types, traffic volumes and weather changes. Therefore, ATCO's have to deal with more and more information that could cause a significant increase in their workload. Appropriate interface design in ATM systems can discharge ATCO cognitive loads and enhance SA by facilitating a better match between task demands and cognitive resource (Kaber, Perry, Segall, Mcclernon, \& Iii, 2006). Effective coordination of HCI is crucial to the successful implementation of innovative systems in the MRTO environment. Interface design must apply holistic approaches to facilitate distributed cognition coordination in rapidly changing situations (Langan-Fox, Canty, \& Sankey, 2009), as high performance in conflict detection and resolution has the potential to increase both airspace efficiency and the safety of aviation (Schuster \& Ochieng, 2014). ATCOs' task performance and perceived workload might increase if technologies require operators to process more information and monitor more targets on interface displays. Increased cognitive workloads increase the risk of attentional tunnelling, cognitive lockup, and out-of-the-loop syndrome (Endsley \& Kiris, 1995). One of the most commonly used measures of operator's perceived workload is NASA-TLX (Task Load Index) (Hart \& Staveland, 1988). Workload can negatively affect ATCOs' performance and increase operational errors (Athènes, Averty, Puechmorel, Delahaye, \& Collet, 2002). Wickens (2002) defines workload as the load imposed on the limited information processing resources of the unaided (without assistance of automation) human operator described as the "baseline" or "manual" condition. Task management is directly related to mental workload, as the competing demands of tasks for 
attention exceed the operator's limited resources, and better multitask performance results from rapid switching between tasks (Wickens, 1999).

\section{4 Assessing Human Performance}

Human Error Template (HET) is a formal method to identify human factors issues in the design and certification process in aviation (Stanton, 2006). The method consists of a checklist approach and comes in the form of an error template. HET works as a simple checklist and is applied to each bottom level task step in a Hierarchical Task Analysis (HTA). The technique works by indicating which of the error modes are credible for each task step, based upon the judgement of the analysis participants. The participant simply applies each of the HET error modes to the task step in question and determines whether any of the modes produce any credible errors (Stanton et al., 2008). The strengths of the HET tool are that it is simple to learn and use, it requires very little training and it is designed to be very quick to use. The only drawback of HET is that the process can be tedious when dealing with a large amount of collected data (Stanton et al., 2010). HTA is the analysis of how a task is accomplished by detailed descriptions of both manual and mental activities, task and element durations, task frequency, task allocation, task complexity, environmental conditions, and equipment involved in one or more people to perform a given task. HTA is used to produce an exhaustive description of tasks in a hierarchical structure of goals, sub-goals, operations and plans. The participant then has to determine the probability of the error (low, medium or high) and the criticality of the error (low, medium or high). If the human error is marked as high for both probability and criticality, the operational step involved in the task performance is then rated as a 'concern' requiring intervention (Kearney, Li, Braithwaite, \& Greaves, 2017).

\section{Method}




\subsection{Participants}

Five subject-matter experts, all qualified remote tower controllers participated in this research. The ages of participants were between 41 and 53 years old $(\mathrm{M}=47.2, \mathrm{SD}=4.5)$. The working experience of participants was between 13 and 25 years $(M=17, S D=5.9)$. The approval of the Ethic Committee was granted (CURES/1506/2016) in advance of the research taking place.

\subsection{Apparatus}

The controller working position on the Remote Tower Module is equipped with (1) the OTW displays consisted with fourteen active screens and one standby unit in the event of equipment failure. The displays match the PTZ camera resolution of 1920 x 1080 pixels with a refresh rate of $60 \mathrm{~Hz}$ in a 220 degrees configuration with an infra-red camera; (2) EFS system which is divided into two parts; one for Shannon airport and one for Cork airport; (3) RDP which can be used as a distance indicator to touch-down and is divided into two parts one for Shannon and one for Cork airport; (4) a VCS which was equipped with a Schmid Communications Panel. To increase ATCO's SA, the borders of the display systems of EFS, RDP and OTW were distinguished by colours, purple indicated Shannon and green indicated Cork airport (figure 2).

[Figure 2 here]

\subsection{Task Decomposition of MRTO}

Six focus group sessions were held, and participants were supplied with the HTA and HET methodology which consisted of the step-by-step descriptions of task decomposition, taxonomy modes of human errors, a flowchart showing how to conduct an analysis using the method, an example of an analysis carried out using the method and an example output of the 
HET format. Participants were also given a HTA describing the action stages involved in both remotely controlling a commercial aircraft landing at Shannon airport and simultaneously controlling another aircraft departing from Cork airport from RTC located at Dublin airport. The participants were also provided with access to the MRTO module located at Dublin airport to remotely shadowing control Shannon and Cork airports simultaneously for validating timing and task breakdown of HTA. HTA apply detailed description of the operational actions to achieve the goals (Stanton, 2006; Stanton et al., 2008). The task decomposition involves the breakdown of a task of simultaneously controlling two aircraft located at different airports. It attempts to explain how to achieve the goal of safety by completing each operational action and the time needed to complete each activity. The specific time needed to complete each step allows the assessment of the the criticality of time-limited situations for multiple task performance. ATCO's attention distributions has to shift between airports and tasks to maintain safety of operations.

\subsection{Research Design}

This project applied Saab's remote tower systems consisting of a camera array, pan/tilt/zoom cameras and signal light guns at Cork and Shannon airports controlled from a RTC at Dublin airport. The RTC is equipped with Out the Window visualisation, an electronic flight strip system and an air/ground and ground/ground voice communication system for the appropriate Cork and Shannon VHF frequencies and sector coordination functions respectively. Five subject-matter experts, all ATCOs, applied HTA to break down the scenario into operational steps. ATCO's operational behaviour and their interaction with the various controller working position components including VCS, EFS, OTW, RDP, and PTZ were analysed. The HTA operational steps for simultaneous departing and arriving aircraft from two different airports, including time (in seconds) to complete the tasks, were then integrated with the twelve error modes of HET for criticality analysis (low, medium and high) and probability (low, medium 
and high). Participants had to determine the likelihood of the error and the criticality of the error for each individual operational step of HTA. If the error mode is given a high rating for both probability and criticality, then it is rated as a 'concern', meaning that it requires attention in order to assure and improve safety. If the error mode is given a low rating for both probability and criticality, it will be marked as 'Pass' (Stanton et al., 2017). Pass is defined as an error whose effects would not endanger safety (scores between 1 and 4). Conversely, Concern is defined as those errors where there was a high probability of occurrence and have the potential to endanger safety (scores between 6 and 9). 'Concern' highlighted design issues (hardware, software or operational procedures) which could lead to critical human factors accidents/incidents. These concerns should prompt the designer/regulator to consider changes to, or redesign of interfaces, procedures, and/or ATCO training, in order to eliminate or mitigate the impact of these errors during MRTO.

NASA-TLX was applied to evaluate ATCOs' perceived workload between MRTO and physical tower operations. The high density of traffic and dynamic aircraft manoeuvres in terminal airspace will increase ATCO's perceived workload, as controllers face additional challenges which may decrease controller's performance and create safety concerns. NASATLX is a popular technique for measuring subjective perceived workload including Mental demand, Physical demand, Temporal demand, Performance, Effort and Frustration. The participants were required to evaluate their perceived workload between MRTO and single tower operations after each trial. By analysing these six dimensions, it is possible to understand the various safety concerns in relation to perceived workload and task performance.

\section{Results and Discussions}

MRTO was performed by a single ATCO simultaneously providing ATS to both Shannon and Cork airports. The integration of PTZ with OTW augmented visualization reinforced by RDP 
and EFS technology provided the necessary technical supports for the provision of ATS remotely. The existing data links and communications network delivered the required information without any degradation to the standard of ATS. HTA and HET were conducted at the MRTO centre at Dublin airport to allow participants validate the applicability of the operational steps involved. Furthermore, NASA-TLX was applied to evaluate ATCOs' subjective workload at the end of task completion. The objective of this research is to understand the limitations of HCI and safety concerns related to human performance on MRTO.

\subsection{Task Analysis of Single Controller Performing MRTO}

The task under analysis is 'one ATCO safely controlling a commercial aircraft landing at Shannon airport while simultaneously controlling another commercial aircraft departing from Cork airport from a RTC situated at Dublin airport. In order to distinguish the actions between Cork airport and Shannon airport colour coding was used, green colour represents operational steps related to Cork, and the purple colour represents operational steps related to Shannon. The use of augmented visualization via PTZ operation is a new HCI issue and its impact on task performance and perceived workload does not exist in traditional physical towers (Marchitto, Benedetto, Baccino, \& Canas, 2016). Once the overall task goal of performing MRTO was specified, the next step was to break the overall goal down into meaningful subgoals (Stanton et al., 2004). In the task, "simultaneously Landing at Shannon airport and Departing from Cork airport", the overall goal was broken down into 51 sub-goals. There are 51 operational steps for ATCOs to complete including 27 actions associated with the landing at Shannon and 24 actions associated with the departure from Cork (figure 3). All 51 operational steps must be assessed based on the twelve error modes of HET to identify potential human errors related to MRTO. The overall goal of the task was broken down into the sub-goals. The bottom level of any branch in a HTA should always be an operational 
action(Stanton et al., 2017). Within the sub-goals for one ATCO performing "Simultaneously Landing at Shannon airport and Departing from Cork airport", there were 51 bottom level operational actions to be completed within a time window of 262 seconds. Each operational action contains lots of challenging cognitive and physical demands, these task demands may increase ATCO's perceived workload and decrease performance.

[Figure 3 here]

\subsection{Analysis of Human Performance on MRTO}

The HET matrix was constructed with the vertical-axis assigned as 'likelihood', while the error 'criticality' index was placed on the horizontal-axis. Likelihood and criticality were combined through a multiplication process (likelihood x criticality) to give a 'Pass' or 'Concern' of predicting error related to HCI design on MRTO. A condition determined through the HET to have achieved a likelihood and criticality combination between 1 and 4 was assigned as 'Pass', a score between 6 and 9 classified as 'Concern'. An example of operational step 1.2.4 Scan of Shannon airport OTW and RDP is shown as table 1.

[Table 1 here]

The majority of operational steps are marked as PASS with medium likelihood and low criticality. Only two error modes raised safety concerns with HET for MRTO, these are completion of a runway scan prior to a runway operation with an associated concern of task executed incomplete and Scan of Shannon airport OTW and RDP in five seconds with an associated concern of misread of information (table 1). These two concerns, task execution incomplete, raised a concern (score 9) with a high likelihood for incomplete scan of Shannon airport's runway (score 3), with high criticality of runway incursion (score 3); and misread information (score 6) for medium likelihood (score 2) of scanning without paying attention 
with high criticality of runway incursion (score 3). Furthermore, the time frame of each operational step identified in the HTA is under normal operations, it is likely that should a critical event occur or an unusual or abnormal pilot request to ATC occur, there is potential for workload to increase and time pressure to become more acute. The operational steps shown in purple are related to Shannon airport, the green is related to Cork airport in the figure 3. Each operational step was expected to be finished within a time frame. Though the majority of operational steps are marked as PASS with medium likelihood and low criticality, some of these, such as task repeat on scan Shannon runway was time consuming leading to task executed too late and reducing SA, these also increased ATCO workload as some steps require crosschecking to assure safety.

The results of HTA and HET demonstrate that advanced technology integrated with augmented visualization (PTZ, OTW, RDP and EFS) design improved ATCO's monitoring performance for controlling aircraft from two airports simultaneously. A fundamental principle for the introduction of any new technology is that it must first achieve at least the same level of safety of ATS provision as that which is provided using the traditional physical tower. The analysis of human performance by HET can provide the evidence, arguments and assumptions to support this principle. During the trials the ATCOs and the RTC project team were governed by the same safety management policies, principles and procedures that exist for Local Tower operations. There were no safety occurrences during the trials, albeit there were some safety concerns due to time pressure and the prioritisation of operational steps for multiple tasks. An important technical requirement is that the visual presentation of aircraft and vehicles by the remote tower system shall not exceed the 1,000 milliseconds of end-toend delay in order to fit the requirements of safety assessment. There is a requirement for further research on ATCO's visual behaviours related to human performance on MRTO (European Aviation Safety Agency, 2015). 


\subsection{ATCO’s Perceived Workload on MRTO}

ATCO's cognitive processes and performance has been the subject of much concentration in research and practice including SA, decision-making, mental workload, and operational performance. However, there are lots of arguments for finding strong empirical evidence and lacking scientific status. The high density of traffic and dynamic aircraft manoeuvres in the terminal airspace will increase ATCO's perceived workload, as controllers face additional difficulties which will decrease controller's performance and create safety concerns. The results of ATCOs' subjective perceived workload between single tower and MRTO by NASA-TLX is demonstrated in table 2. The test of normality for paired samples' differences was verified by using Shapiro-Wilk test, and the results showed that all six dimensions of NASA-TLX do not go against normal distribution $(\mathrm{p}>0.05)$. Therefore, a paired T-test could be applied to analyze the differences in the six dimensions of NASA-TLX. The results demonstrated that there were significant differences for ATCO mental demand ( $\mathrm{t}=2.955, \mathrm{p}=0.006, \mathrm{~d}=0.540)$, temporal demand $(\mathrm{t}=12.181, \mathrm{p}<0.001, \mathrm{~d}=2.224)$, effort $(\mathrm{t}=14.203, \mathrm{p}<0.001, \mathrm{~d}=2.593)$ and frustration $(\mathrm{t}=14.050, \mathrm{p}<0.001, \mathrm{~d}=2.565)$ between MRTO and physical tower operations. However, there were no significant differences between ATCO physical demand $(t=1.510, p=0.142)$ and performance $(\mathrm{t}=-1.044, \mathrm{p}=0.3055)$ between MRTO and physical tower operations (figure 4$)$. MRTO operational tasks require involved more moving targets and more monitoring tasks than a physical tower operation, this means the ATCO must work harder to maintain safe separations in MRTO operations, this can induce additional pressure on ATCOs which may lead to them experience stress, fatigue and annoyance (Cao, Chintamani, Pandya, \& Ellis, 2009). These may explain the significant higher mental demand, temporal demand, effort and frustration scores on MRTO than single tower operation. MRTO ATCOs have to maintain the same level of performance to ensure the safety of operations but at the cost of higher cognitive loads instead of physical demand. Previous research demonstrated that low workload had a negative influence on performance as it can aggravate boredom, and high workload can result in poor 
performance due to stress or overload (Eggemeier, 1988); however, high workload might provoke a strategy shift so that the operators perform well (Moehlenbrink, Papenfuss, \& Jakobi, 2012). The high density of traffic and dynamic aircraft manoeuvres in terminal airspace will increase ATCO's perceived workload. This research found that NASA-TLX may reflect different components of workload including 6 dimensions and it may not co-vary with measures of all aspects of performance (Hart, 2006).

[Table 2 here]

[Figure 4 here]

The results demonstrated the concept of MRTO is applicable to provide both air and ground movement controls for two low volume airports. However, how much is too much when it comes to tasks for a single controller? MRTO is safe whilst operations are routine, the evolution of a critical event at one or two airports has the potential to overload the single ATCO, this requires additional study and analysis before MRTO operations can be deployed (Kearney \& $\mathrm{Li}, 2018$ ). The development of new remote tower technology is designed to reduce ATCO's workload through augmented vision presented on OTW, RDP and EFS. However, the added complexity of multiple tasks did create more cognitive loads to ATCOs to process huge volumes of information (Wiener, 1988; Li, Kearney, Braithwaite, \& Lin, 2018). Augmented visualization design of RTM allows ATCOs to change the size of the screen for selected airports, this innovative technology has significantly increased ATCO's task performance. However, there are also some potential risks related to $\mathrm{HCI}$ and human performance and this requires further research to precisely identify these impacts and suggest suitable mitigation strategies to defend against the risks (Ltifi, Kolski, \& Ayed, 2015).

\section{Conclusion}


The application of remote tower technologies can assist ANSP's achieve cost efficiency and safety requirements as mandated through the EU Single European Sky project. This research demonstrated that augmented visualization using panorama video cameras did provide sufficient technical support for a single ATCO to perform tasks initially planned to be achieved by four ATCOs, however, the demands of multiple tasks also induced significant workload. It must be stated that this research is based on normal operations and does not consider the impact of an unusual situation, critical event or emergency during the operation. Should an unexpected event occur, it is reasonable to expect that workload will likely increase thus having the potential to negatively impact on ATCO's performance. This creates a need for further research on how to relieve ATCO's workload. MRTO has been proven as safe as the local tower operation in providing ATS. The novelty and flexibility of remote tower technology may allow regulators to be creative in adapting safety regulations and ANSP's to be more operationally agile in the management of varying traffic volumes. Nevertheless, the evolution and deployment of MRTO systems requires a cautious balance between cost-efficiency and the potential impacts on safety, capacity, and human performance.

\section{Acknowledgment}

The authors would like to express special thanks to the Irish Aviation Authority for their support in funding this research, and also to the ATCOs who took part in the research. Their support, enthusiasm and engagement are invaluable in facilitating the authors' research efforts.

\section{References}

Ahlstrom, U., \& Friedman-Berg, F. (2006). Using eye movement activity as a correlate of cognitive workload. International Journal of Industrial Ergonomics, 36(7), 623-636. doi:10.1016/j.ergon.2006.04.002 
Athènes, S., Averty, P., Puechmorel, S., Delahaye, D., \& Collet, C. (2002). ATC complexity and controller workload: Trying to bridge the gap. In Proceedings of the International Conference on HCI in Aeronautics (pp. 56-60). AAAI Press Cambridge, MA.

Beier, K., \& Gemperlein, H. (2004). Simulation of infrared detection range at fog conditions for Enhanced Vision Systems in civil aviation. Aerospace Science and Technology, 8(1), 63-71. doi:10.1016/j.ast.2003.09.002

Cao, A., Chintamani, K. K., Pandya, A. K., \& Ellis, R. D. (2009). NASA TLX: Software for assessing subjective mental workload. Behavior research methods, 41(1), 113-117. doi: 10.3758/BRM.41.1.113

Durso, F. T., Truitt, T. R., Hackworth, C. A., Crutchfield, J. M., \& Manning, C. A. (1998). En route operational errors and situational awareness. The International Journal of Aviation Psychology,8(2), 177-194. doi: 10.1207/s15327108ijap0802_6

Eggemeier, F. T. (1988). Properties of workload assessment techniques. Human mental workload, 52, 41-62. doi: 10.1016/S0166-4115(08)62382-1

Endsley, M. R. (1995). Measurement of situation awareness in dynamic systems. Human Factors, 37(1), 65-84. doi: 10.1518/001872095779049499

Endsley, M. R., \& Kiris, E. O. (1995). The out-of-the-loop performance problem and level of control in automation. Human factors, 37(2), 381-394. doi:

$10.1518 / 001872095779064555$

Eurocontrol (2014). Eurocontrol Seven-year IFR Flight Movements and Service Units Forecast: 2014-2020 (Reference No. 14/02/24-43). Brussels, Belgium. 
outlook. Brussels, Belgium.

European Aviation Safety Agency. (2015). Guidance material on the implementation of the remote tower concept for single mode of operation.

Fürstenau, N., Mittendorf, M., \& Friedrich, M. (2016). Model-Based Analysis of TwoAlternative Decision Errors in a Videopanorama-Based Remote Tower Work Position. In Virtual and Remote Control Tower (pp. 241-260). Springer, Cham. doi: 10.1007/978-3-319-28719-5_11

Fürstenau, N., \& Schmidt, M. (2016). Remote Tower Experimental System with Augmented Vision Videopanorama. In Virtual and Remote Control Tower (pp. 163-192): Springer, Cham. doi: 10.1007/978-3-319-28719-5_8

Hart, S. G. (2006). NASA-task load index (NASA-TLX); 20 years later. Paper presented at the Proceedings of the human factors and ergonomics society annual meeting. doi: $10.1177 / 154193120605000909$

Hart, S. G., \& Staveland, L. E. (1988). Development of NASA-TLX (Task Load Index): Results of empirical and theoretical research. Advances in psychology, 52, 139-183. doi: $10.1016 / \mathrm{S} 0166-4115(08) 62386-9$

Hollan, J., Hutchins, E., \& Kirsh, D. (2000). Distributed cognition: toward a new foundation for human-computer interaction research. ACM Transactions on Computer-Human Interaction (TOCHI), 7(2), 174-196. doi: 10.1145/353485.353487

Kaber, D. B., Perry, C. M., Segall, N., Mcclernon, C. K., \& Iii, L. (2006). Situation awareness implications of adaptive automation for information processing in an air traffic control-related task. International Journal of Industrial Ergonomics, 36(5), 447-462. 
doi: 10.1016/j.ergon.2006.01.008

Kearney, P. \& Li, W-C. (2018). Multiple Remote Tower for Single European Sky: the Evolution from Initial Operational Concept to Regulatory Approved Implementation. Transportation Research Part-A, 116, 15-30. doi: 10.1016/j.tra.2018.06.005

Kearney, P., Li, W-C., Braithwaite, G., \& Greaves, M. (2017). The Investigation HumanComputer Interaction on Multiple Remote Tower Operations. In Proceedings of 2017International Conference on Human Computer Interaction. Vancouver, Canada. doi: 10.1007/978-3-319-58472-0_23

Komogortsev, O. V., \& Karpov, A. (2013). Automated classification and scoring of smooth pursuit eye movements in the presence of fixations and saccades. Behavior Research Methods, 45(1), 203-215. doi:10.3758/s13428-012-0234-9

Lafond, D., Champagne, J., Hervet, G., Gagnon, J. F., Tremblay, S.,\& Rousseau, R. (2009). Decision Analysis Using Policy Capturing and Process Tracing Techniques in a Simulated Naval Air-Defense Task. Proceedings of the Human Factors and Ergonomics Society Annual Meeting, 53(18), 1220-1224. doi:

$10.1177 / 154193120905301812$

Langan-Fox, J., Canty, J. M., \& Sankey, M. (2009). Human-automation teams and adaptable control for future air traffic management. International Journal of Industrial Ergonomics, 39(5), 894-903. doi: 10.1016/j.ergon.2009.04.002

Laois, L., \& Giannacourou, M. (1995). Perceived effects of advanced ATC functions on human activities: Results of a survey on controllers and experts. Proceedings of the Eighth International Symposium on Aviation Psychology, 2, 392-397.

Li, W-C., Kearney, P., Braithwaite, G. R., \& Lin, J. (2018). How much is too much on monitoring tasks? Visual scan patterns of single air traffic controller performing 
multiple remote tower operations. International Journal of Industrial Ergonomics, 67, 135-144. doi: 10.1016/j.ergon.2018.05.005

Ltifi, H., Kolski, C., \& Ayed, M. B. (2015). Combination of cognitive and HCI modeling for the design of KDD-based DSS used in dynamic situations. Decision Support Systems, 78, 51-64. doi: 10.1016/j.dss.2015.07.003

Marchitto, M., Benedetto, S., Baccino, T., \& Canas, J. (2016). Air traffic control: Ocular metrics reflect cognitive complexity. International Journal of Industrial Ergonomics, 54, 120-130. doi:10.1016/j.ergon.2016.05.010

Moehlenbrink, C., Papenfuss, A., \& Jakobi, J. (2012). The role of workload for work organization in a remote tower control center. Air Traffic Control Quarterly, 20(1), 526. doi: 10.2514 /atcq.20.1.5

Pinska, E. (2006). An investigation of the head-up time at tower and ground control positions. Proceedings 5th Eurocontrol Innovative Research Workshop, 81-86.

Schmidt, M., Rudolph, M., \& Fürstenau, N. (2016). Remote Tower Prototype System and Automation Perspectives. Virtual and Remote Control Tower (pp. 193-220): Springer. doi: 10.1007/978-3-319-28719-5_9

Schuster, W., \& Ochieng, W. (2014). Performance requirements of future Trajectory Prediction and Conflict Detection and Resolution tools within SESAR and NextGen: Framework for the derivation and discussion. Journal of Air Transport Management,35(4), 92-101. doi: 10.1016/j.jairtraman.2013.11.005

Stanton, N. A. (2006). Hierarchical task analysis: Developments, applications, and extensions. Applied ergonomics, 37(1), 55-79. doi: 10.1016/j.apergo.2005.06.003 
Stanton, N. A., Harris, D., Salmon, P. M., Demagalski, J., Marshall, A., Waldmann, T., . . \& \& Young, M. S. (2010). Predicting design-induced error in the cockpit. Journal of aeronautics, astronautics and aviation, 42(1), 1-10. doi: 10.6125/JAAA

Stanton, N. A., Hedge, A., Brookhuis, K., Salas, E., \& Hendrick, H. W. (2004). Handbook of human factors and ergonomics methods. CRC press.

Stanton, N. A., Salmon, P., Harris, D., Marshall, A., Demagalski, J., Young, M. S., . . \& Dekker, S. (2008). Predicting pilot error on the flight deck: Validation of a new methodology and a multiple methods and analysts approach to enhancing error prediction sensitivity.

Stanton, N. A., Salmon, P. M., Rafferty, L. A., Walker, G. H., Baber, C., \& Jenkins, D. P. (2017). Human factors methods: a practical guide for engineering and design. CRC Press.

Tobaruela, G., Schuster, W., Majumdar, A., Ochieng, W. Y., Martinez, L., \& Hendrickx, P. (2014). A method to estimate air traffic controller mental workload based on traffic clearances. Journal of Air Transport Management, 39, 59-71. doi:

10.1016/j.jairtraman.2014.04.002

Wickens, C. D. (1999). Frames of reference for navigation. In D. Gopher \& A. Koriat (Eds.), Attention and performance. Attention and performance XVII: Cognitive regulation of performance: Interaction of theory and application (pp. 113-144). Cambridge, MA, US: The MIT Press.

Wickens, C. (2002). Multiple resources and performance prediction. Theoretical issues in ergonomics science, 3(2), 159-177. doi: 10.1080/14639220210123806 
Wickens, C., \& Hollands, J. (2000). Signal Detection, Information Theory, and Absolute Judgment. Engineering psychology and human performance, 2, 24-73.

Wiener, N. (1988). The human use of human beings: Cybernetics and society (NO. 320) Perseus Books Group.

Yu, C-S., Li, W-C., Wang, E. M., Braithwaite, G., \& Greaves, M. (2016). Pilots' visual scan patterns and attention distribution during the pursuit of a dynamic target. Aerospace Medicine and Human Performance, 87(1), 40-47. doi:10.3357/AMHP.4209.2016 
Table 1. Example of HET output on Scan OTW and RDP on Multiple Remote Tower Operations

\begin{tabular}{|c|c|c|c|c|c|c|c|c|c|c|c|}
\hline \multicolumn{3}{|c|}{$\begin{array}{l}\text { Scenario: Simultaneously Landing on EINN and } \\
\text { Departing on EICK }\end{array}$} & \multicolumn{9}{|c|}{ Task step: 1.2.4 Scan of EINN OTW + RDP (5 seconds) } \\
\hline \multirow[b]{2}{*}{ Error Mode } & \multirow[b]{2}{*}{ TICK } & \multirow[b]{2}{*}{ Description } & \multirow[b]{2}{*}{ Outcome } & \multicolumn{3}{|c|}{ Likelihood } & \multicolumn{3}{|c|}{\begin{tabular}{|l} 
Criticality \\
\end{tabular}} & \multirow{2}{*}{ 胥 } & \multirow{2}{*}{ 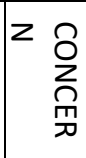 } \\
\hline & & & & $\begin{array}{l}\mathrm{H} \\
3\end{array}$ & $\begin{array}{l}M \\
2\end{array}$ & $\begin{array}{l}\mathrm{L} \\
1\end{array}$ & $\begin{array}{l}\mathrm{H} \\
3\end{array}$ & $\begin{array}{l}M \\
2\end{array}$ & $\begin{array}{l}\mathrm{L} \\
1\end{array}$ & & \\
\hline Fail to execute & v & No check on EINN & $\begin{array}{ll}\begin{array}{l}\text { Possible } \\
\text { incursion }\end{array} & \text { Runway } \\
\end{array}$ & & V & & & V & & V & \\
\hline $\begin{array}{l}\text { Task execution } \\
\text { incomplete }\end{array}$ & v & $\begin{array}{l}\text { Incomplete scan of } \\
\text { the Runway }\end{array}$ & $\begin{array}{l}\text { Possible Runway } \\
\text { incursion }\end{array}$ & $\mathrm{v}$ & & & $\mathrm{v}$ & & & & v \\
\hline \multicolumn{12}{|l|}{$\begin{array}{l}\text { Task executed in } \\
\text { wrong direction }\end{array}$} \\
\hline $\begin{array}{l}\text { Wrong task } \\
\text { executed }\end{array}$ & v & $\begin{array}{l}\text { Scanning Cork } \\
\text { thinking it is Shannon }\end{array}$ & $\begin{array}{l}\text { Possible Runway } \\
\text { incursion }\end{array}$ & & V & & & V & & V & \\
\hline Task repeated & v & $\begin{array}{l}\text { Repeated scan of } \\
\text { EINN }\end{array}$ & Time consuming & & & v & & & V & V & \\
\hline $\begin{array}{l}\text { Task executed } \\
\text { on wrong } \\
\text { interface } \\
\text { element }\end{array}$ & V & $\begin{array}{l}\text { Scanning Cork } \\
\text { thinking it is Shannon }\end{array}$ & $\begin{array}{l}\text { Possible Runway } \\
\text { incursion }\end{array}$ & & V & & & V & & V & \\
\hline $\begin{array}{l}\text { Task executed } \\
\text { too early }\end{array}$ & V & $\begin{array}{l}\text { Scanning of Shannon } \\
\text { is done at an early } \\
\text { stage }\end{array}$ & $\begin{array}{l}\text { Increased workload } \\
\text { as subsequent scans } \\
\text { will be carried out }\end{array}$ & & & $\mathrm{v}$ & & & V & V & \\
\hline $\begin{array}{l}\text { Task executed } \\
\text { too late }\end{array}$ & V & $\begin{array}{l}\text { Scanning of Shannon } \\
\text { is done at a later stage }\end{array}$ & $\begin{array}{l}\text { Delayed situational } \\
\text { awareness }\end{array}$ & & & $\mathrm{V}$ & & & V & V & \\
\hline $\begin{array}{l}\text { Task executed } \\
\text { too much }\end{array}$ & V & $\begin{array}{l}\text { Repeated scan of } \\
\text { EINN }\end{array}$ & Time consuming & & & $\mathrm{V}$ & & & V & V & \\
\hline $\begin{array}{l}\text { Task executed } \\
\text { too little }\end{array}$ & V & $\begin{array}{l}\text { Incomplete scan of } \\
\text { the Runway }\end{array}$ & $\begin{array}{l}\text { Possible Runway } \\
\text { incursion }\end{array}$ & & V & & & V & & V & \\
\hline $\begin{array}{l}\text { Misread } \\
\text { information }\end{array}$ & V & $\begin{array}{l}\text { Scanning without } \\
\text { paying attention }\end{array}$ & $\begin{array}{ll}\begin{array}{l}\text { Possible } \\
\text { incursion }\end{array} & \text { Runway } \\
\end{array}$ & & V & & $\mathrm{v}$ & & & & V \\
\hline $\begin{array}{l}\text { Other (extra } \\
\text { unexpected } \\
\text { calls) }\end{array}$ & & $\begin{array}{l}\text {...if increasing } \\
\text { workload, the } \\
\text { likelihood of certain } \\
\text { error modes may } \\
\text { increase as well }\end{array}$ & $\begin{array}{l}\text { depending on the } \\
\text { error type, feed in } \\
\text { turn into the } \\
\text { criticality of the } \\
\text { error... }\end{array}$ & & V & & & V & & & \\
\hline
\end{tabular}


Table 2. T-Test of six dimensions of NASA-TLX between multiple remote tower operations (MRTO) and single physical tower operations

\begin{tabular}{|c|c|c|c|c|c|c|c|c|c|}
\hline \multirow[b]{2}{*}{ Dimensions } & \multirow[b]{2}{*}{ Towers } & \multirow[b]{2}{*}{ Mean } & \multirow[b]{2}{*}{ SD } & \multirow[b]{2}{*}{$\mathrm{N}$} & \multicolumn{5}{|l|}{ T-Test } \\
\hline & & & & & $\mathrm{t}$ & df & $\mathrm{p}$ & SE & $\begin{array}{l}\text { Cohen's } \\
\text { d }\end{array}$ \\
\hline \multirow[b]{2}{*}{$\begin{array}{l}\text { Mental } \\
\text { demand }\end{array}$} & MRTO & 46.833 & 9.781 & & & & & & \\
\hline & $\begin{array}{l}\text { Single } \\
\text { tower }\end{array}$ & 37.833 & 13.814 & 30 & 2.955 & 29 & 0.006 & 3.046 & 0.540 \\
\hline \multirow{2}{*}{$\begin{array}{l}\text { Physical } \\
\text { demand }\end{array}$} & MRTO & 31.667 & 7.350 & & & & & & \\
\hline & $\begin{array}{l}\text { Single } \\
\text { tower }\end{array}$ & 28.500 & 7.673 & 30 & 1.510 & 29 & 0.142 & 2.097 & 0.276 \\
\hline \multirow{2}{*}{$\begin{array}{l}\text { Temporal } \\
\text { demand }\end{array}$} & MRTO & 74.333 & 17.157 & & & & & & \\
\hline & $\begin{array}{l}\text { Single } \\
\text { tower }\end{array}$ & 33.833 & 14.779 & 30 & 12.181 & 29 & 0.000 & 3.325 & 2.224 \\
\hline \multirow[b]{2}{*}{ Performance } & MRTO & 85.000 & 3.714 & & & & & & \\
\hline & $\begin{array}{l}\text { Single } \\
\text { tower }\end{array}$ & 86.667 & 7.112 & 30 & -1.044 & 29 & 0.305 & 1.596 & -0.191 \\
\hline \multirow[b]{2}{*}{ Effort } & MRTO & 84.167 & 5.884 & & & & & & \\
\hline & $\begin{array}{l}\text { Single } \\
\text { tower }\end{array}$ & 35.333 & 17.066 & 30 & 14.203 & 29 & 0.000 & 3.438 & 2.593 \\
\hline \multirow[b]{2}{*}{ Frustration } & MRTO & 71.167 & 18.321 & & & & & & \\
\hline & $\begin{array}{l}\text { Single } \\
\text { tower }\end{array}$ & 22.000 & 13.038 & 30 & 14.050 & 29 & 0.000 & 3.499 & 2.565 \\
\hline
\end{tabular}

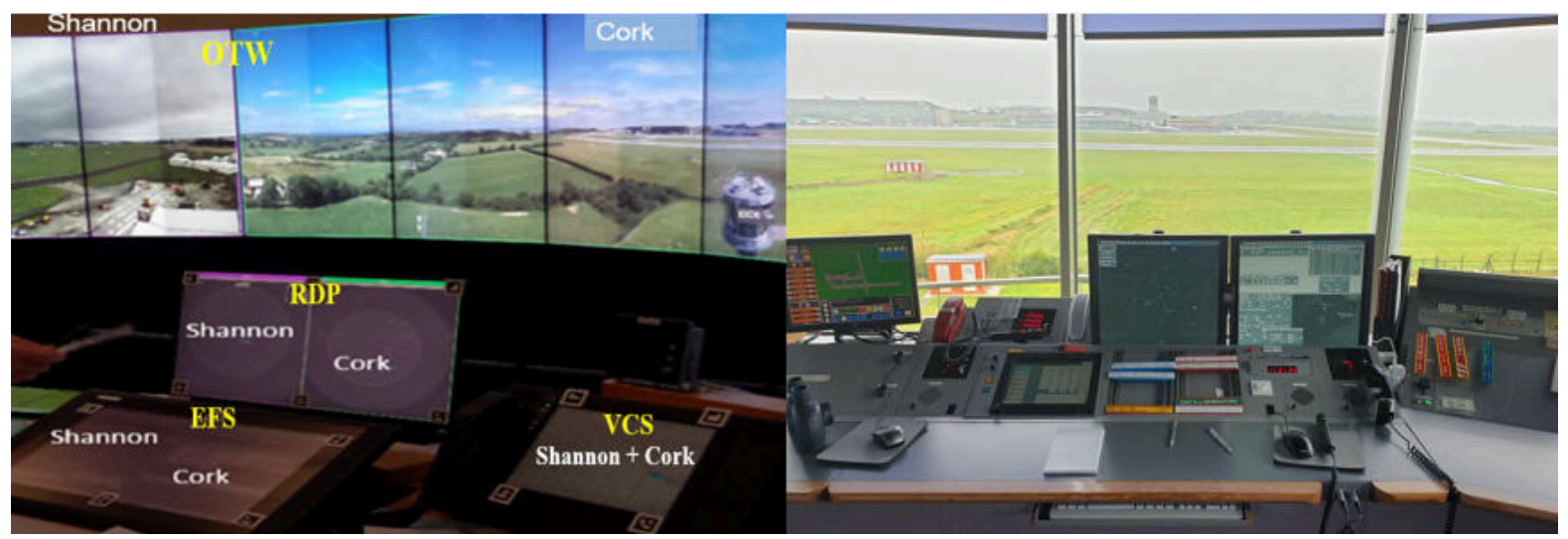

Figure 1. The Controller Working Position consisted by OTW, RDP, EFS and VCS for both Shannon and Cork airports located at Dublin airport on Multiple Remote Tower Operations (left) compared with the layout of traditional physical tower (right) 


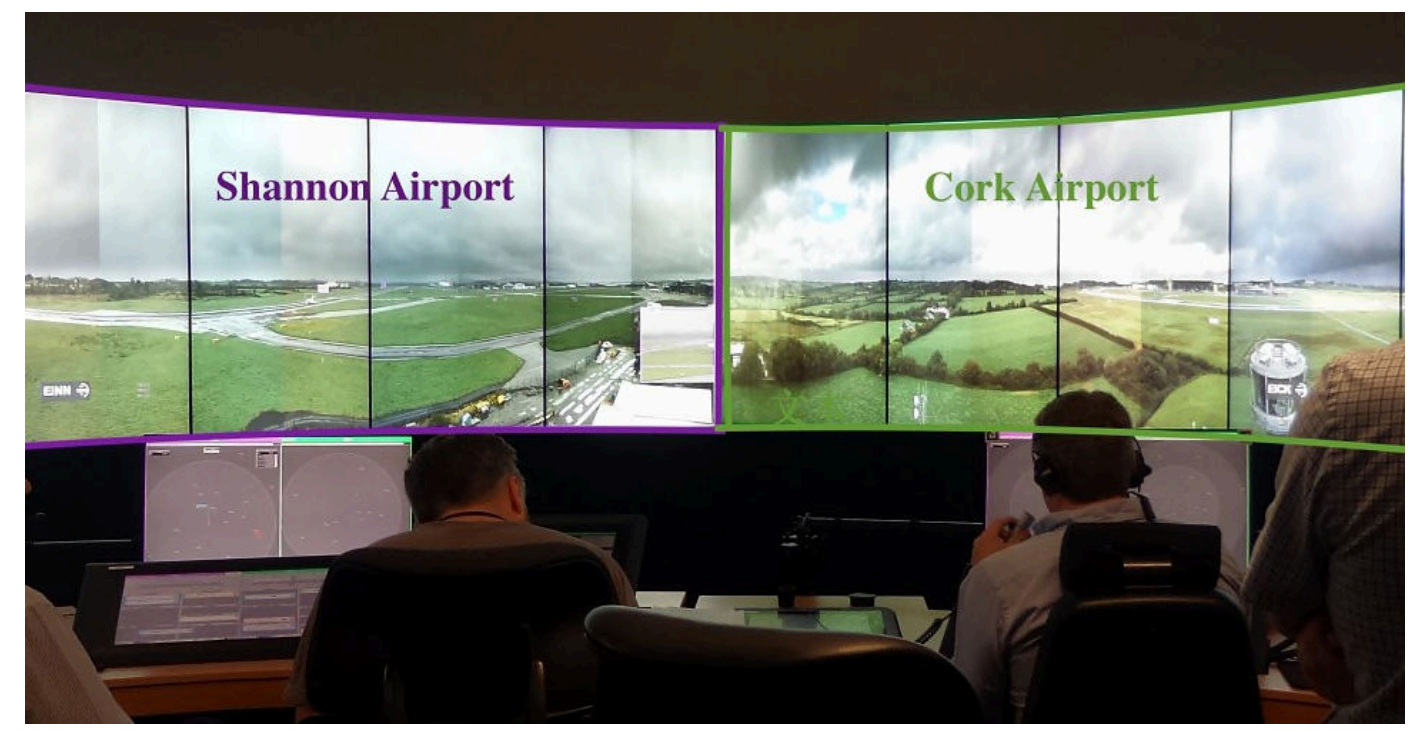

Figure 2. The augmented vision of out of window view for multiple remote tower operations including infra cameras, pan tilt zoom and radar information for different airports shown by different colours on the boarder of displays (Green for Cork airport, Purple for Shannon airport) 


\section{Landing on EINN and Departing on EICK}

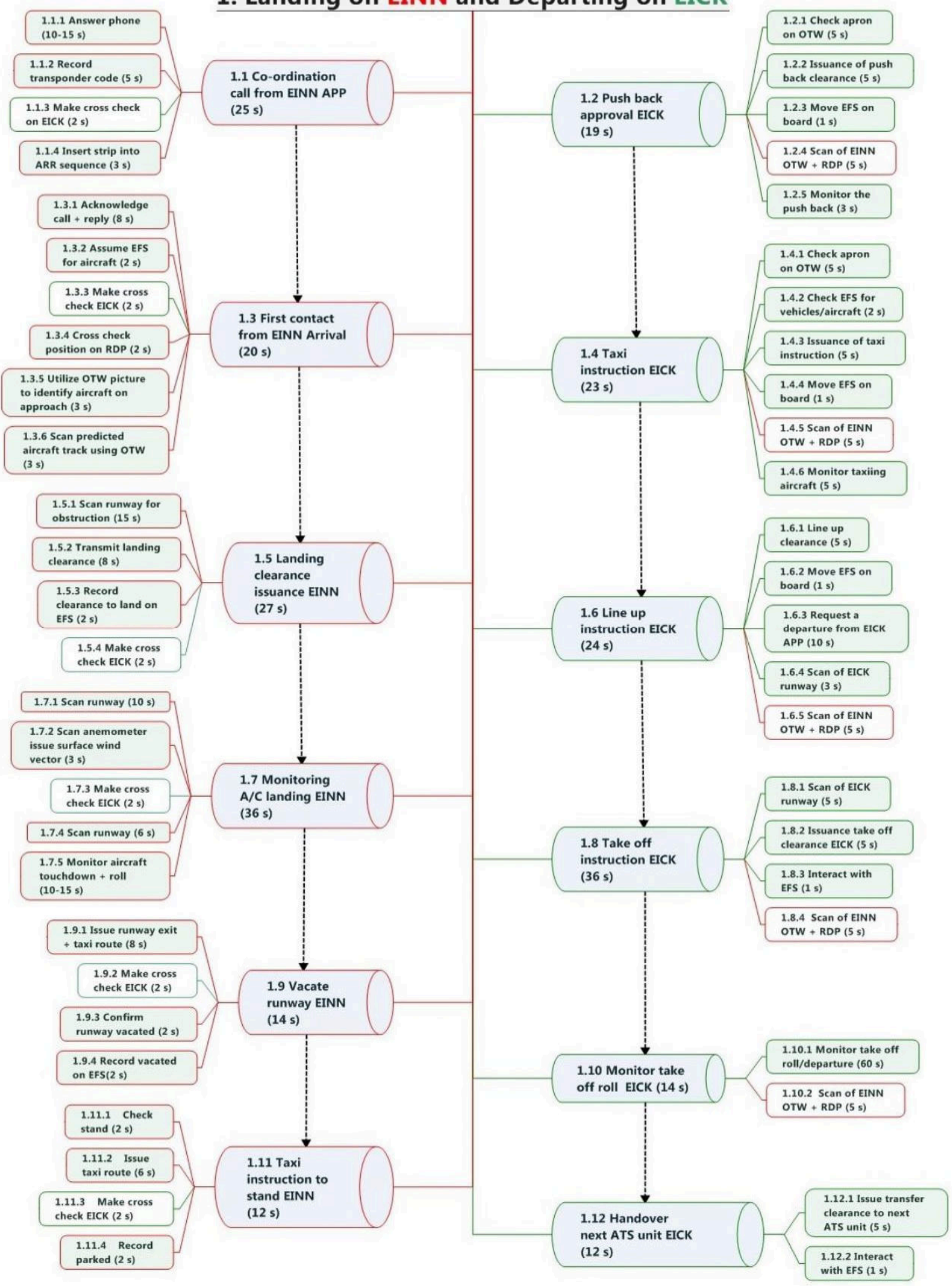

Figure 3. HTA of MRTO for one ATCO control two commercial aircraft simultaneously landing on Shannon and departing from Cork 


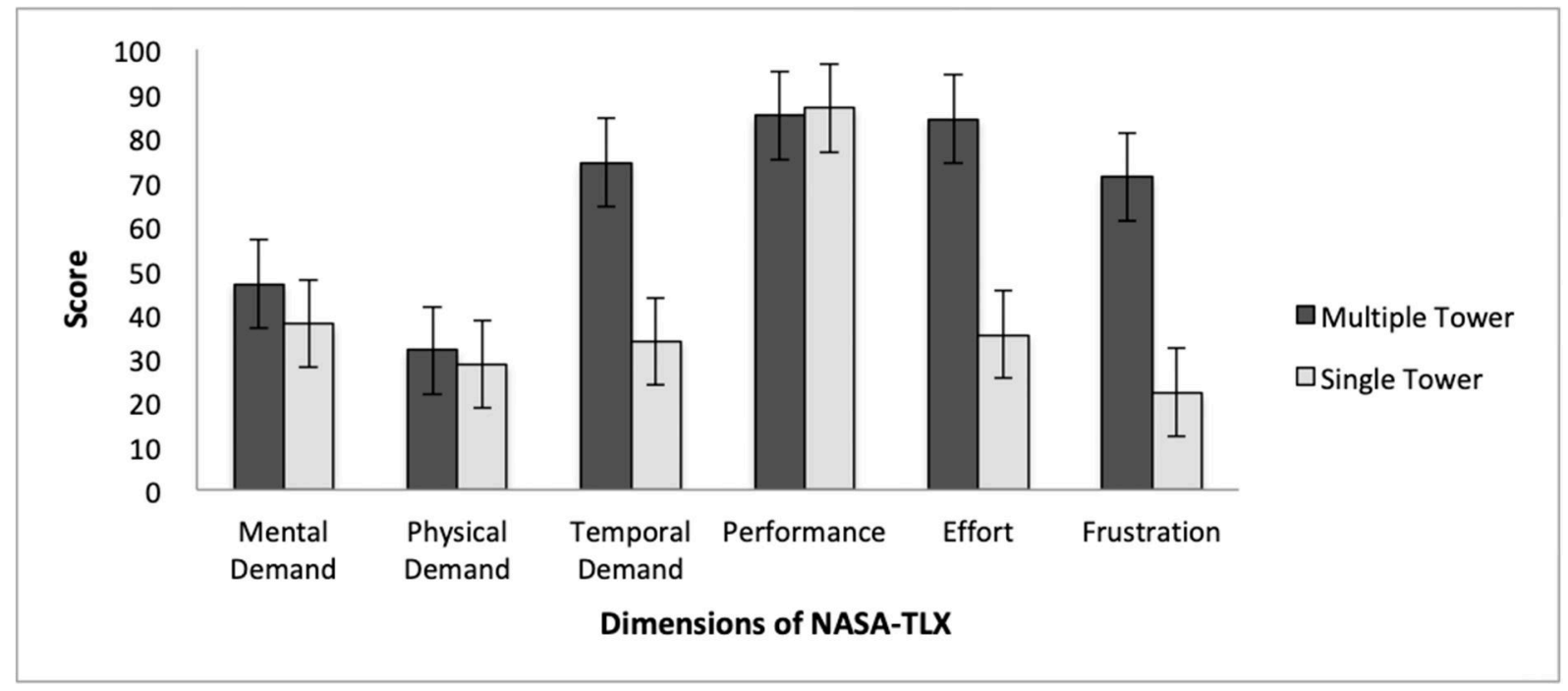

Figure 4. The comparison of perceived workload between single tower operation and multiple remote tower operations 
2019-11-13

Human performance assessment of a single air traffic controller conducting multiple remote tower operations

Kearney, Peter

Wiley

Kearney P, Li W-C, Zhang J, et al., (2020) Human performance assessment of a single air traffic controller conducting multiple remote tower operations. Human Factors and Ergonomics in Manufacturing and Service Industries, Volume 30, Issue 2, March 2020, pp.114-123

https://doi.org/10.1002/hfm.20827

Downloaded from Cranfield Library Services E-Repository 